\title{
Effectiveness of the ATM Forum source traffic description
}

\author{
S. Galmés \\ Universitat de les Illes Balears
}

Ctra. de Valldemossa, km. 7. 5, 07071 Palma, Spain, Tel: + 34-71172989,Fax:+34-71-173003,E-mail:dmisgo0@ps.uib.es

H. G. Perros

North Carolina State University

Department of Computer Science, and Center for Communications and Signal Processing, NCSU, Raleigh, NC 27695, USA

R. Puigjaner

Universitat de les Illes Balears

Ctra. de Valldemossa, km. 7. 5, 07071 Palma, Spain, Tel: + 34-71173288,Fax:+34-71-173003,E-mail:putxi@ps.uib.es

\begin{abstract}
Source traffic description proposed by the ATM Forum is intended to simplify the specification of the intrinsic traffic characteristics at the UNI level. However, from the point of view of the internal traffic management, this description can be inadequate, since it ignores important factors such as burstiness and correlation. In this paper, we make this fact clear in an analytical way by considering on-off processes with arbitrary distributions. For these processes, we provide exact closed-form expressions such as the squared coefficient of variation (a measure of burstiness) and the autocorrelation of the successive inter-arrival times.
\end{abstract}

User Network Interface (UNI)

Keywords

\section{INTRODUCTION}

Early ATM standardization process, carried out by the ATM Forum, has established three traffic descriptors, as part of the connection traffic description, to characterize the cell stream generated by a source (Gün, 1993). As it is shown in (Gün, 1993), these source traffic 
descripturs are une peak cell rate, the sustainable cell rate and the burst tolerance. Along with its simplicity, this characterization facilitates the design of an effective policing mechanism, the Generic Cell Rate Algorithm (GCRA), which is based on a leaky bucket operation (de Pricker, 1991). The three descriptors determine, by means of a very simple relation given in (Gün, 1993), the maximum length of a compliant burst (the longest burst capable of flowing through the policing algorithm without dropping any cell). Since this maximum length is more intuitive than the burst tolerance, and the sustainable cell rate is really an average rate (more specifically, it is a GCRA-oriented average rate definition), we can use from now on the peak rate, the average rate and the maximum burst length to describe the ATM source traffic.

From another point of view, this traffic description is inadequate, since it does not capture completely the notions of burstiness and correlation of the cell stream. It is well known that both characteristics can greatly influence the quality of service offered by the network to its users (Hong, 1992), (You, 1992), (Park, 1992). Then, it turns out that a connection admission control procedure based only on those three descriptors is fairly inefficient. Particularly, we model the cell stream as an on-off process with independent arbitrary distributions; it is becoming apparent that this model constitutes a quite good representation of the future ATM traffic (Perros, 1992), mainly in the case of bursty data applications. Then, we end up with exact closed-form expressions for the burstiness and the correlation of this model, which show that different source traffics may present the same three descriptors and, at the same time, they may behave quite differently from the point of view of burstiness and correlation.

In Section 2 of this paper, we consider the characterization of a generic on-off model according to the ATM Forum traffic description. In Section 3, we obtain closed-form expressions of the squared coefficient of variation (our measure of burstiness) and the correlation of the interarrival times. To end up with these expressions, we apply some results of Discrete Renewal Theory (Taylor, 1984) (in fact, the on-off model is constituted by two alternating renewal processes). Our results show that the on-off model captures completely the notion of burstiness, and even the notion of correlation, despite the independence assumptions. They also show that we can easily play with some parameters of the distributions to modify both burstiness and correlation, but keeping the peak rate, the average rate and the maximum burst length constant. In other words, a call admission control procedure designed around these three descriptors will be transparent to some relevant factors that affect the general performance of the ATM network. In Section 4, we introduce some numerical results, for which we have considered particular distributions of the on and off periods. In this context, some interesting conclusions of the VISTAnet experiment have been applied (Holtsinger, 1993). Finally, in Section 5, we describe the main conclusions of this paper and we provide ideas for further research.

We continue in this paper the analysis performed in (Galmés, 1994). Specifically, we apply the theoretical formulation introduced in (Galmés, 1994) to compute the correlation of a generic on-off traffic model; then, from the analytical expressions of burstiness and correlation, we focus into the source traffic description of the ATM Forum.

\section{TRAFFIC DESCRIPTION OF THE ON-OFF FLUID MODEL}

Let's consider an on-off traffic model whose active (on) and silence (off) periods have independent arbitrary distributions. We can describe the duration of both periods by means of two discrete independent random variables, $a$ for the active period and $s$ for the silence period. Each variable is characterized by a probability function $\left(p_{a}, p_{s}\right)$ and a cumulative distribution function $\left(F_{a}, F_{s}\right)$. Usually, both variables take values within some given ranges:

$p_{a}(k)>0$ for $k \in[m b l, M B L] ; p_{a}(k)=0$ for $k \notin[m b l, M B L] \quad ;$ 
$p_{s}(k)>0$ for $k \in[m s p, M S P] ; p_{s}(k)=0$ for $k \notin[m s p, M S P]$

where $m b l$ is the minimum length of the active period and $M B L$ is its maximum length. Similarly, $m s p$ is the minimum silence period and $M S P$ is the maximum silence period.

Then, the average length of both types of periods can be easily expressed in this way:

$$
\begin{aligned}
& \bar{a}=\sum_{k=m b l}^{M B L} k p_{a}(k) ; \\
& \bar{s}=\sum_{k=m s p}^{M S P} k p_{s}(k) .
\end{aligned}
$$

The traffic so far modelled will show these three ATM descriptors (Gün, 1993), (de Pricker, 1991):

a. Average rate $(m)$, expressed in cells per slot:

$m=\frac{\bar{a}}{\bar{a}+\bar{s}}$.

b. Peak rate $\left(R_{p}\right)$ :

$R_{p}=1$,

since in our definition each active period has 1 cell per slot.

c. The maximum burst length is already provided by the model $(M B L)$.

\section{BURSTINESS AND CORRELATION OF THE ON-OFF TRAFFIC MODEL}

Let's adopt the squared coefficient of variation of the interarrival times as the measure of burstiness. Thus, both parameters, burstiness and correlation, involve the interarrival time between subsequent cells. Let $T_{n}$ be the interarrival time between the $n$th cell and the $(n+1)$ th cell, as we show in Figure 1.

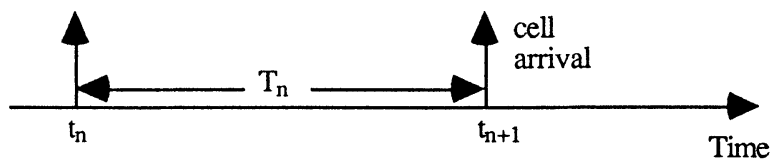

Figure 1 Definition of the interarrival time between adjacent cells.

For the expression of $T_{n}$, there are two possibilities to deal with:

1. The instant $t_{n}$ corresponds to the arrival of the last cell of an active period. Then, this cell is followed by a silence period, as we show in Figure 2(a). Consequently, the interarrival time obeys this expression: 
$T_{n}=1+s$.

2. The instant $t_{n}$ corresponds to an intermediate cell of an active period. In this case, according to Figure 2(b), the interarrival time is 1:

$T_{n}=1$.

Since all active (burst) periods are equally distributed and independent, they constitute a renewal process and we can apply some important results of Discrete Renewal Theory (Taylor, 1984); particularly, it is specially interesting for us the residual life of an active period at a given instant of time (the observation instant), which is assumed to correspond to the arrival of a cell. Then, let $\hat{a}$ be the residual life of an active period at the arrival of an arbitrary cell. From (Taylor, 1984), we can set up this expression for the long run behaviour of the probability function $\widehat{p_{a}}$ of this new random variable:

$\widehat{p_{a}}(k)=\frac{1-F_{a}(k-1)}{\bar{a}}$,
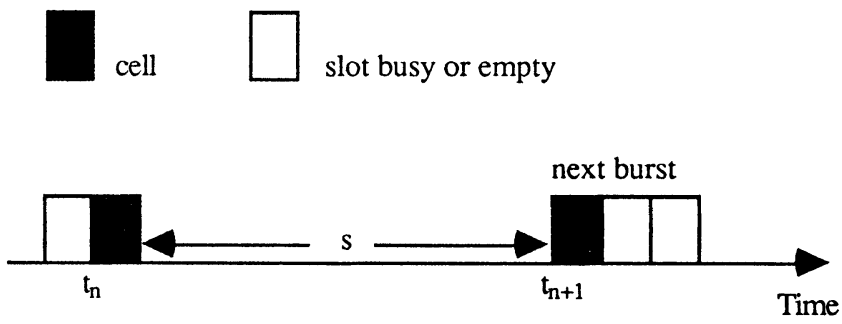

(a)

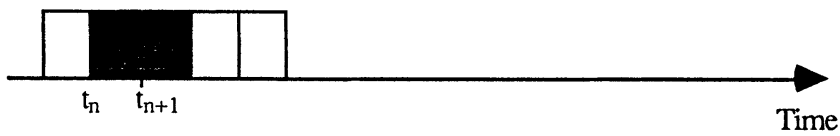

(b)

Figure 2 Interarrival time: (a) when subsequent cells belong to different bursts; (b) when they belong to the same burst.

where

$k \in[1, M B L]$.

The variable $k$ takes the value of 1 when we make the observation at the arrival of the last cell of a burst period; in the other extreme, it is equal to the maximum burst length when we make the observation at the beginning of a burst period of maximum duration.

As it is stated in (Taylor, 1984), expression (5) holds if 1 is the greatest common divisor of the integers for which the probability function $p_{a}$ is strictly positive. There is no need for any algebra to assure that this condition will be easily satisfied. For example, if one of the 
integers is prime, unless all the other numbers are multiple of it (very uncommon), the condition is verified.

As we mentioned before, expression (5) characterizes the long run behaviour of the probability function of the residual life. Then, from a theoretical point of view, it "never" takes place; however, from a practical point of view, we can generally make use of the asymptotic formulation after some initial (transient) period with an acceptable accuracy.

Next, on the basis of the renewal formulation, we end up with closed-form expressions for the burstiness and the correlation of the model we have introduced:

a) Burstiness. A good measure of burstiness for any traffic model is the squared coefficient of variation of the successive interarrival times $\left(c^{2}\right)$ :

$b=c^{2}=\frac{\operatorname{var}\left(T_{n}\right)}{\overline{T_{n}^{2}}}$

where $b$ denotes the degree of burstiness.

This burstiness can be analytically expressed by making use of the Discrete Renewal Theory results described before. As far as the expected interarrival time is concerned, it should give us the inverse of the average rate. We can check it:

$$
\begin{aligned}
& \overline{T_{n}}=1 \cdot \operatorname{prob}(\hat{\mathrm{a}} \geq 2)+\sum_{s=m s p}^{M S P}(1+s) \operatorname{prob}(\hat{\mathrm{a}}=1, s)= \\
& =\sum_{\hat{\mathrm{a}}=2}^{M B L} \widehat{p_{a}}(\hat{\mathrm{a}})+\sum_{s=m s p}^{M S P}(1+s) \widehat{p_{a}}(1) p_{s}(s)= \\
& =1-\widehat{p_{a}}(1)+\widehat{p_{a}}(1) \sum_{s=m s p}^{M S P}(1+s) p_{s}(s)= \\
& =1-\widehat{p_{a}}(1)+(1+\bar{s}) \widehat{p_{a}}(1)=1+\bar{s} \widehat{p_{a}}(1)=1+\frac{\bar{s}}{\bar{a}}=\frac{1}{m} .
\end{aligned}
$$

This analysis is based on expressions (3), (4a), (4b) and (5) and the assumption of independence between active and silence periods. The fact we have got the true result for the expected interarrival time enforces the correctness of using the residual life distribution.

The same considerations are involved when we compute an expression for the variance of the interarrival times:

$$
\begin{aligned}
& \operatorname{var}\left(T_{n}\right)=\overline{T_{n}^{2}}-\overline{T_{n}}{ }^{2} ; \\
& \overline{T_{n}^{2}}=1^{2} \cdot \operatorname{prob}(\hat{\mathrm{a}} \geq 2)+\sum_{s=m s p}^{M S P}(1+s)^{2} \operatorname{prob}(\hat{\mathrm{a}}=1, s)= \\
& =1-\widehat{p_{a}}(1)+\sum_{s=m s p}^{M S P}(1+s)^{2} \widehat{p_{a}}(1) p_{s}(s)= \\
& =1-\widehat{p_{a}}(1)+\widehat{p_{a}}(1) \sum_{s=m s p}^{M S P}(1+s)^{2} p_{s}(s)=
\end{aligned}
$$




$$
\begin{aligned}
& =1-\widehat{p_{a}}(1)+\widehat{p_{a}}(1) \sum_{s=m s p}^{M S P}\left(1+2 s+s^{2}\right) p_{s}(s)= \\
& =1-\widehat{p_{a}}(1)+\widehat{p_{a}}(1)\left[1+2 \bar{s}+\operatorname{var}(s)+\bar{s}^{2}\right]= \\
& =1+\frac{\bar{s}}{a}\left[2+\left(1+c_{s}^{2}\right) \bar{s}\right]= \\
& =1+\frac{1-m}{m}\left[2+\left(1+c_{s}^{2}\right) \bar{s}\right],
\end{aligned}
$$

where $c_{s}^{2}$ is the squared coefficient of variation of the silence periods.

Then,

$$
\operatorname{var}\left(T_{n}\right)=1+\frac{1-m}{m}\left[2+\left(1+c_{s}^{2}\right) \bar{s}\right]-\frac{1}{m^{2}}=\frac{1-m}{m}\left[\left(1+c_{s}^{2}\right) \bar{s}-\frac{1-m}{m}\right]
$$

and, finally, we end up with the expression of burstiness:

$b=\frac{\frac{1-m}{m}\left[\left(1+c_{s}^{2}\right) \bar{s}-\frac{1-m}{m}\right]}{\frac{1}{m^{2}}}=m(1-m)\left[\left(1+c_{s}^{2}\right) \bar{s}-\frac{1-m}{m}\right]$.

This is an interesting result, since it shows that the burstiness of a cell stream modelled as an on-off process with independent arbitrary distributions hardly depends on the shape of these distributions; it just depends on the first two moments. More exactly, it depends on the first moment of the distribution of the active periods (through the average rate), and the first and second moments of the distribution of the silence periods.

It also clearly shows that we can modify the value of burstiness by varying the average length of the silence periods or their squared coefficient of variation, and keeping constant, at the same time, the traffic descriptors established by the ATM Forum. In Figure 3 we provide graphic representations of the relationships between burstiness and the three variables appeared in (9).

b) Correlation between successive interarrival times. The fact that our traffic is modelled as the alternation of two independent renewal processes does not avoid, surprisingly, a correlation between successive interarrival times. The lag-1 correlation obeys this expression:

$\rho(1)=\frac{\overline{T_{n} T_{n+1}}-\overline{T_{n}}}{\operatorname{var}\left(T_{n}\right)}$.

Table 1 Basic combinations of the values of two successive interarrival times

\begin{tabular}{ll}
\hline $\mathrm{T}_{\mathrm{n}}$ & $\mathrm{T}_{\mathrm{n}+1}$ \\
\hline 1 & 1 \\
1 & $1+\mathrm{s}$ \\
$1+\mathrm{s}$ & 1 \\
$1+\mathrm{s}$ & $1+\mathrm{s}^{\prime}$ \\
\hline
\end{tabular}


It just remains for us to determine the first term of the numerator of (10). We have, based again on expressions (4a) and (4b), four basic combinations of the values of the successive interarrival times to deal with. We show these combinations in Table 1 and their graphic representation in Figure 4. In the first case, the three cells considered belong to the same burst; in the second case, two adjacent cells complete one burst and the third cell initiates a new burst; the third case is like the second one, but in the reverse order; finally, in the last case, one cell completes a burst, the next cell constitutes a burst by itself and the third cell begins a new burst (in this case, we have to use two variables to denote both off periods).

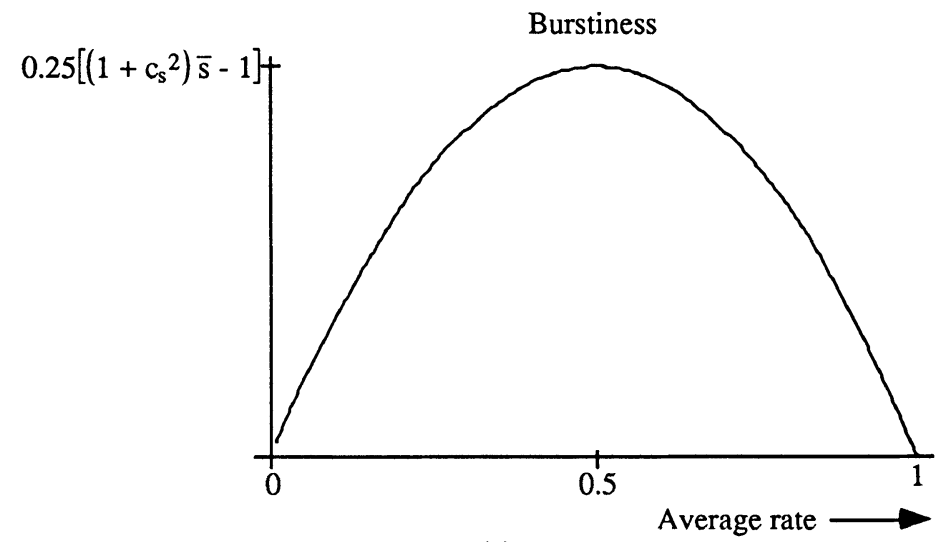

(a)

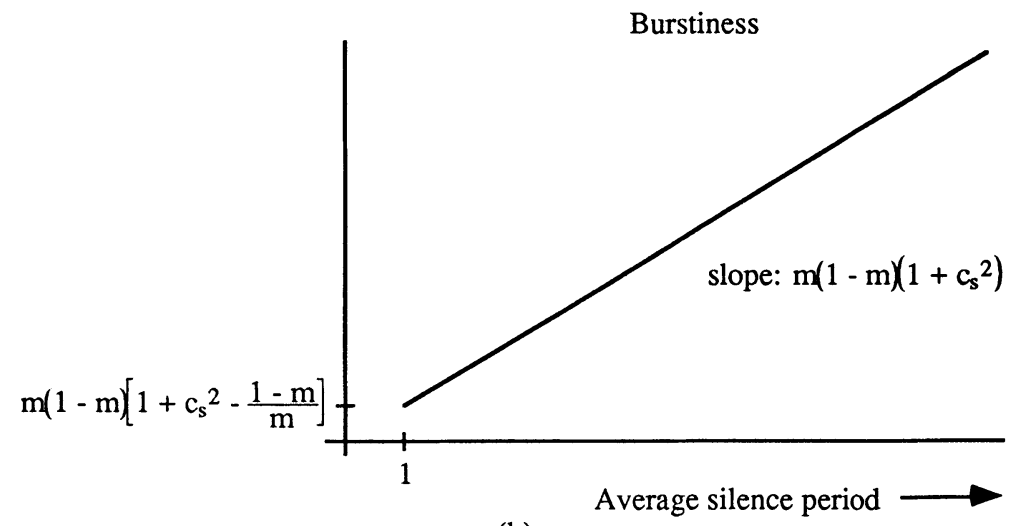

(b) 


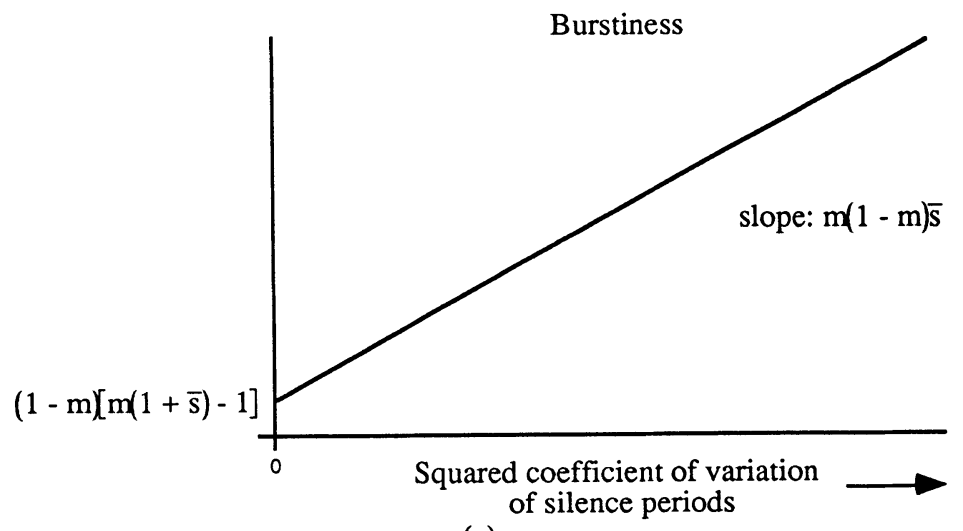

(c)

Figure 3 Burstiness of an on-off process as a function of: (a) the average rate; (b) the average length of silence periods; (c) the squared coefficient of variation of silence periods.

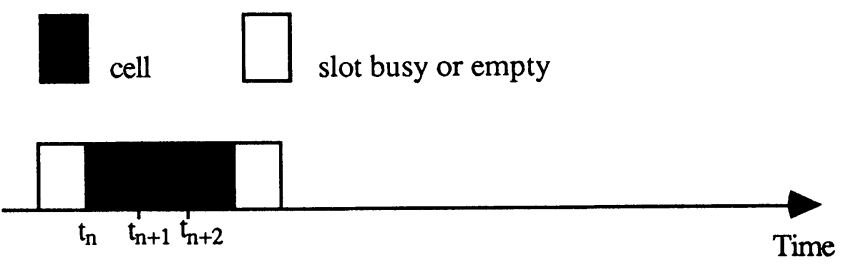

(a)

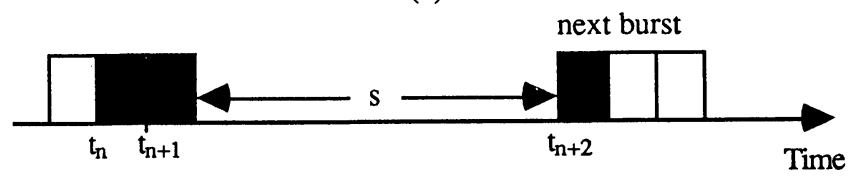

(b)

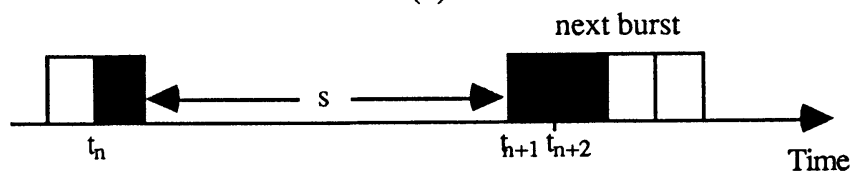

(c) 


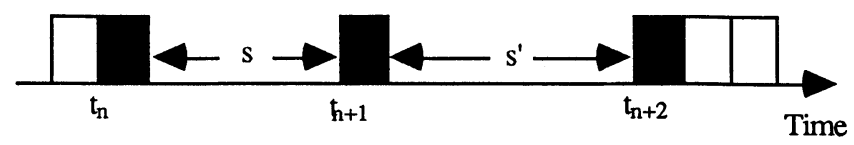

(d)

Figure 4 Illustration of the four combinations described in table 1: (a) $T_{n}=1$ and $T_{n+1}=1$; (b) $T_{n}=1$ and $T_{n+1}=1+s$; (c) $T_{n}=1+s$ and $T_{n+1}=1$; (d) $T_{n}=1+s$ and $T_{n+1}=1+s^{\prime}$.

So, using again the distribution of the residual time given in (5), we can obtain an expression for the expected value of the product between successive interarrival times:

$$
\begin{aligned}
& \overline{T_{n} T_{n+1}}=1 \cdot 1 \cdot \operatorname{prob}(\hat{\mathrm{a}} \geq 3)+\sum_{s=m s p}^{M S P} 1 \cdot(1+s) \operatorname{prob}(\hat{\mathrm{a}}=2, s)+ \\
& +\sum_{s=m s p}^{M S P}(1+s) \cdot 1 \cdot \operatorname{prob}(\hat{\mathrm{a}}=1, s, a \geq 2)+ \\
& +\sum_{s=m s p, s^{\prime}=m s p}^{M S P, M S P}(1+s)\left(1+s^{\prime}\right) \operatorname{prob}\left(\hat{\mathrm{a}}=1, s, a=1, s^{\prime}\right)= \\
& =\sum_{\hat{a}=3}^{M B L} \widehat{p_{a}}(\hat{\mathrm{a}})+\sum_{s=m s p}^{M S P}(1+s) \widehat{p_{a}}(2) p_{s}(s)+ \\
& +\sum_{s=m s p}^{M S P}(1+s) \widehat{p_{a}}(1) p_{s}(s) \sum_{a=2}^{M B L} p_{a}(a)+ \\
& +\sum_{s=m s p}^{M S P} \sum_{s^{\prime}=m s p}^{M S P}(1+s)\left(1+s^{\prime}\right) \widehat{p_{a}}(1) p_{s}(s) p_{a}(1) p_{s}\left(s^{\prime}\right)= \\
& =1-\widehat{p_{a}}(1)-\widehat{p_{a}}(2)+\widehat{p_{a}}(2) \sum_{s=m s p}^{M S P}(1+s) p_{s}(s)+ \\
& +\widehat{p_{a}}(1) \sum_{a=2}^{M B L} p_{a}(a) \sum_{s=m s p}^{M S P}(1+s) p_{s}(s)+ \\
& +p_{a}(1) \widehat{p_{a}}(1) \sum_{s=m s p}^{M S P}(1+s) p_{s}(s) \sum_{s^{\prime}=m s p}^{M S P}\left(1+s^{\prime}\right) p_{s}\left(s^{\prime}\right)= \\
& =1-\widehat{p_{a}}(1)-\widehat{p_{a}}(2)+\widehat{p_{a}}(2)(1+\bar{s})+\widehat{p_{a}}(1)\left[1-p_{a}(1)\right](1+\bar{s})+p_{a}(1) \widehat{p_{a}}(1)(1+\bar{s})^{2}= \\
& =1-\widehat{p_{a}}(1)-\widehat{p_{a}}(2)+\widehat{p_{a}}(2)+\widehat{p_{a}}(2) \bar{s}+\widehat{p_{a}}(1)(1+\bar{s})+p_{a}(1) \widehat{p_{a}}(1)(1+\bar{s}) \bar{s}= \\
& =1+\bar{s}\left[\widehat{p_{a}}(1)+\widehat{p_{a}}(2)\right]+p_{a}(1) \widehat{p_{a}}(1)(1+\bar{s}) \bar{s}= \\
& =1+\bar{s}\left[\frac{1}{\bar{a}}+\frac{1-p_{a}(1)}{\bar{a}}\right]+p_{a}(1)(1+\bar{s}) \frac{\bar{s}}{\bar{a}}= \\
& =1+\bar{s}\left[\frac{1}{\bar{a}}+\frac{1-p_{a}(1)}{\bar{a}}+\frac{p_{a}(1)}{\bar{a}}(1+\bar{s})\right]=
\end{aligned}
$$




$$
\begin{aligned}
& =1+\left[\frac{2}{\bar{a}}+p_{a}(1) \frac{\bar{s}}{\bar{a}}\right] \bar{s}=1+\frac{\bar{s}}{\bar{a}}\left[2+\bar{s} p_{a}(1)\right]= \\
& =1+\frac{1-m}{m}\left[2+\bar{s} p_{a}(1)\right] .
\end{aligned}
$$
be:

We have also applied here the independence assumptions. Then, the lag-1 correlation will

$\rho_{1}=\frac{1+\frac{1-m}{m}\left[2+\bar{s} p_{a}(1)\right]-\frac{1}{m^{2}}}{\frac{1-m}{m}\left[\left(1+c_{s}^{2}\right) \bar{s}-\frac{1-m}{m}\right]}=\frac{\frac{1-m}{m}\left[\bar{s} p_{a}(1)-\frac{1-m}{m}\right]}{\frac{1-m}{m}\left[\left(1+c_{s}^{2}\right) \bar{s}-\frac{1-m}{m}\right]}=\frac{\bar{s} p_{a}(1)-\frac{1-m}{m}}{\left(1+c_{s}^{2}\right) \bar{s}-\frac{1-m}{m}}$.

So, the Discrete Renewal Theory focus provides also an analytic expression for the correlation between successive interarrival times, which shows that usually this correlation is non-zero, despite all independence hypothesis set up in our modelling approach. It is still possible to rewrite expression (12) in a more simplified way, in which we make clearer that really the correlation does not depend on the average silence period:

$\rho_{1}=\frac{\bar{s} p_{a}(1)-\frac{1-m}{m}}{\left(1+c_{s}^{2}\right) \bar{s}-\frac{1-m}{m}}=\frac{\bar{s} p_{a}(1)-\frac{\bar{s}}{\bar{a}}}{\left(1+c_{s}^{2}\right) \bar{s}-\frac{\bar{s}}{\bar{a}}}=\frac{p_{a}(1)-\frac{1}{\bar{a}}}{1+c_{s}^{2}-\frac{1}{\bar{a}}}$.

Really, this correlation does not depend on the average length of the silence periods. As in the case of burstiness, the shapes of the distributions hardly affect to correlation, just only through the proportion of unitary bursts and the wideness of the silence period distribution. Correlation also depends on the average length of the active periods. Its sign is positive or negative depending on the comparative relation between the unitary burst probability and the inverse of the average length of the active periods. Also, we can introduce a change in correlation through any of the parameters appeared in (13), keeping constant the three traffic descriptors.

In Figure 5, we show the evolution of correlation as a function of the squared coefficient of variation of the silence periods. If this coefficient is slightly high, the correlation is going to be low, for any value of the unitary burst probability. Just in the case of a high unitary burst probability and a low degree of variation of the length of silence periods, we can get an appreciable correlation.

\section{NUMERICAL RESULTS}

It is hard nowadays to predict how ATM traffic will behave in the future, but measures from some experimental ATM networks, like the VISTAnet Gigabit Network (Holtsinger, 1993), point out to on-off traffic models with constant distribution for the active period. As far as the silence period is concerned, uniform, geometric or hyper-geometric distributions seem to be better descriptors. Let's assume a simple on-off traffic model with constant on period and uniform off period. Then,

$p_{a}(k)=1$ for $k=M B L ; p_{a}(k)=0$ for $k \neq M B L$ 


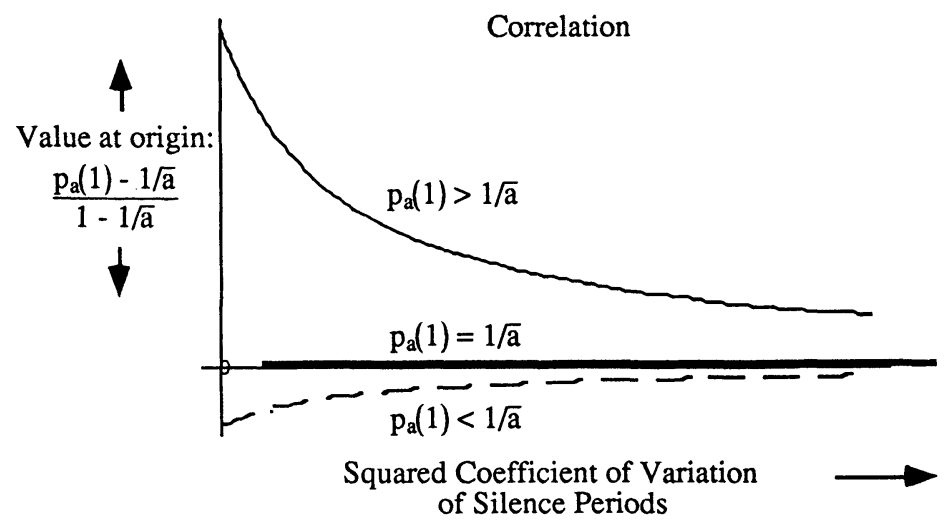

Figure 5 Correlation between successive interarrival times as a function of the squared coefficient of variation of silence periods.

and

$p_{s}(k)=\frac{1}{M S P-m s p+1}$ for $k \in[m s p, M S P] ; p_{s}(k)=0$ for $k \notin[m s p, M S P]$.

The average length of the active period will be, of course, MBL, this is,

$\bar{a}=M B L$.

The expectation, variance and squared coefficient of variation of the uniform distribution of silence periods are also simple:

$\bar{s}=\frac{M S P+m s p}{2} ;$

$\operatorname{var}(s)=\frac{(M S P-m s p+1)^{2}-1}{12} ;$

$c_{s}^{2}=\frac{(M S P-m s p+1)^{2}-1}{3(M S P+m s p)^{2}}$.

Now, we can use these equalities in (9) and (13) to end up with the expressions of burstiness and correlation:

$b=\frac{2 M B L(M S P+m s p)}{(2 M B L+M S P+m s p)^{2}}\left\{\left[1+\frac{(M S P-m s p+1)^{2}-1}{3(M S P+m s p)^{2}}\right]\left(\frac{M S P+m s p}{2}\right)-\frac{M S P+m s p}{2 M B L}\right\} ;$ 


$$
\rho_{1}=-\frac{1}{M B L\left[1+\frac{(M S P-m s p+1)^{2}-1}{3(M S P+m s p)^{2}}\right]-1}
$$

Next, we consider the influence of the width of the uniform distribution on burstiness and correlation for a given value of the average silence period; in other words, we are going to modify the values of MSP and msp in such a way that their sum keeps constant. Then, these changes affect only, through the variance, the squared coefficient of variation of silence periods, but they don't affect the average rate of the traffic flow and the maximum burst length. To make things easy, let's define two new variables:

$\omega=M S P+m s p ;$

$\Delta=M S P-m s p$.

Then, we can rewrite expressions (18) and (19) according to these new definitions:

$$
\begin{aligned}
& b=\frac{2 M B L \cdot \omega}{(2 M B L+\omega)^{2}}\left\{\left[1+\frac{(\Delta+1)^{2}-1}{3 \omega^{2}}\right] \frac{\omega}{2}-\frac{\omega}{2 M B L}\right\} ; \\
& \rho_{1}=-\frac{1}{M B L\left[1+\frac{(\Delta+1)^{2}-1}{3 \omega^{2}}\right]-1} .
\end{aligned}
$$

In our experiment, the maximum value for $\Delta$ is determined by the expected value of the distribution, because any silence period is always equal or longer than 1:

$\Delta_{\max }=2(\bar{s}-1)$.

Assuming an arrival process with $M B L=40$ and $\omega=200$, we show in Figure 6 how the width $\Delta$ affects its behaviour concerning burstiness and correlation.

It is clear from Figure 6 that our particular process is highly uncorrelated for any value of width, but it's bursty, and this burstiness clearly depends on the width of the silence period distribution. From the user-network interface point of view, no distinction would be done among all the processes located within the scope of Figure 6; however, processes with high values of width should cause much more congestion problems and bottlenecks at switching nodes than processes with a narrow distribution of the inactive period. In (Hong, 1992), (You, 1992), (Park, 1992) we can find interesting results that corroborate these arguments.

\section{CONCLUSIONS}

We have shown in this paper that the source traffic description proposed by the ATM Forum is not effective from the point of view of the internal traffic control. The reason is that this description does not match some important traffic features in congestion control and quality of service, such as burstiness and correlation. In the analysis, we have considered on-off traffic models with independent arbitrary distributions and, in addition, we have proposed an interesting mathematical tool such as Discrete Renewal Theory. This tool has let to us to end up with closed-form expressions for burstiness and correlation independently of the particular 
distributions of the active and silence periods. It is specially interesting that, despite the independence assumptions, the correlation is not necessarily zero. Besides, these expressions are relatively simple, since they hardly involve the shape of the distributions. Finally, we
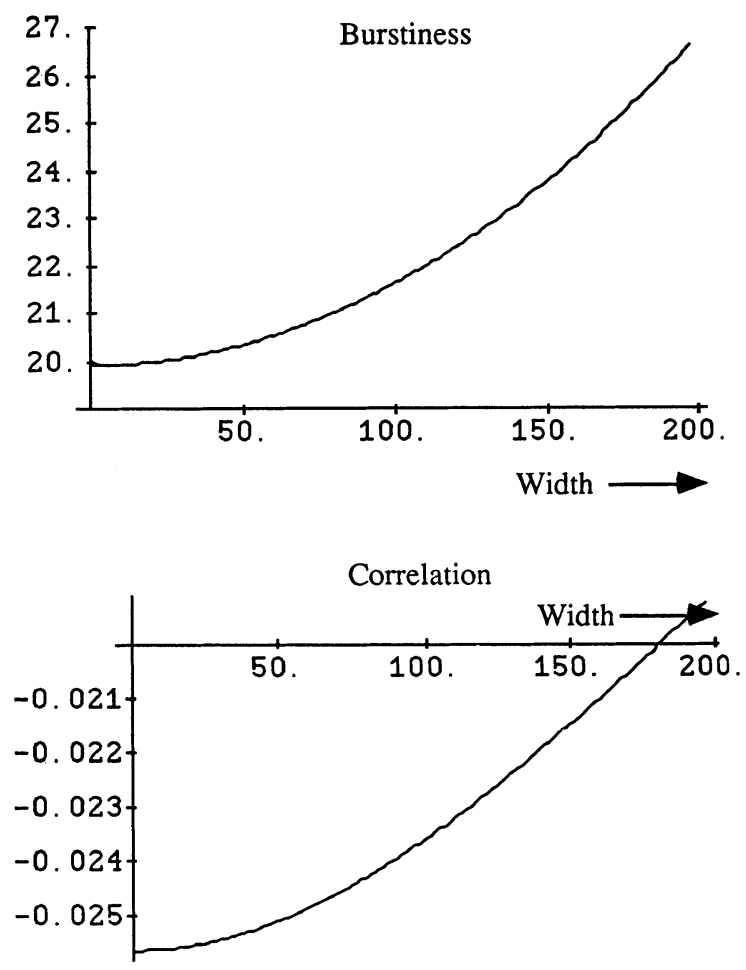

Figure 6 Influence of the width of the silence period distribution on burstiness and correlation of the arrival process. This one is assumed to have a constant active period equal to 40 and a uniform silence period with expectation equal to 100 .

have applied the general results to one particular traffic model, for which we have computed more specific expressions and numerical and graphic results. This model (constant on period and uniformly distributed off period) is quite realistic and, at the same time, due to its simplicity, has clearly shown the theoretical basis of this paper. In summary, we have made clear in this paper a disadvantatge of the source traffic description proposed by the ATM Forum, despite all their advantatges, mentioned in Section 1; this result could be previously guessed, but we have shown it in this paper in a mathematical way on the basis of general onoff sources. At the same time, and even more important, we have illustrated the capability of Discrete Renewal Theory for dealing with this type of models, since it has let us to end up with exact results.

Further research could be addressed to the analysis of the performance parameters of an ATM switch with several on-off input sources. In this context, it could be checked the 
validity of the independence assumptions for describing the superposition process, and if they depend or not on the number of feeder processes. Another question is how to get good estimates of the statistical parameters (squared coefficient of variation, average active and silence periods and the probability of unitary bursts) from real measures, within acceptable confidence intervals and also within acceptable time intervals. Finally, experimental measures in realistic environments would greatly contribute to validate the theoretical basis of this paper, from the traffic modelling approach to the particular results on burstiness and correlation.

\section{REFERENCES}

de Prycker, M. (1991) Asynchronous Transfer Mode. Solution for Broadband ISDN. Ellis Horwood.

Galmés, S. , Perros, H. G. and Puigjaner, R. (1994) Analysis of Burstiness for On-off Traffic Models with Arbitrary Distributions. Proceedings of the Second Workshop on Performance Modelling and Evaluation of ATM Networks, IFIP TC6 Working Group 6. 4 and TC6 Task Force on Performance of Computer Networks.

Gün, L. and Marin, G. A. (1993) An Overview of the ATM Forum and the Traffic Management Activities, in Asynchronous Transfer Mode Networks (ed. Y. Viniotis and R. O. Onvural). Plenum Press, New York.

Holtsinger, D. S. , Perros, H. G. and Nilsson, A. A. (1993) Analysis of Traffic Measurements in the VISTAnet Gigabit Networking Testbed. Technical Report, TR93/11, Department of Computer Science, North Carolina State University.

Hong, S. , Perros, H. G. and Yamashita, H. (1992) A Discrete-time Queueing Model of the Shared Buffer ATM Switch with Bursty Arrivals. Technical Report, TR-92/11, Department of Computer Science, North Carolina State University.

Park, D. , Perros, H. G. and Yamashita, H. (1992) Approximate Analysis of Discrete-time Tandem Queueing Networks with Bursty and Correlated Input Traffic and Customer Loss. Technical Report, TR-92/22, Department of Computer Science, North carolina State University.

Perros, H. (1992) A Literature Review of Call Admission Algorithms. Technical Report, Department of Computer Science and Center for Communications and Signal Processing, North Carolina State University.

Taylor, H. M. and Karlin, S. (1984) An Introduction to Stochastic Modelling. Academic Press, New York.

You, Y. F. , Nilsson, A. A. and Lai, F. (1992) The Upper Bounds for Performance Measures of a Finite Capacity Polling System under ATM Bursty Arrivals. Technical Report, TR-92/14, Department of Computer Science, North Carolina State University.

You, Y. F. , Nilsson, A. A. and Lai, F. (1992) Approximate Analysis of an ATM Switching System with Bursty Arrivals and Finite Capacity. Technical Report, TR-92/15, Department of Computer Science, North Carolina State University.

You, Y. F. , Nilsson, A. A. and Lai, F. (1992) Performance Modelling of a Finite Capacity System with ATM Bursty and Correlated Input Traffic. Technical Report, TR_92/18, Department of Computer Science, North Carolina State University.

Sebastián Galmés received the degree of Telecommunication Engineer from the Polytechnic University of Catalonia (Spain) in 1989. In 1991 he joined the Department of Computer Science of the University of Balearic Islands (Spain), where he is currently Associate Professor of Network Architecture and Queueing Theory and Simulation. During the academic year 1992-93 he was on a sabbatical leave of absence at the Computer Science Department of the North Carolina State University. His research interests are in the area of ATM networking, mainly in traffic modelling and performance evaluation. 
Harry G. Perros received the B. Sc. degree in Mathematics in 1970 from Athens University, Greece, the M. Sc. degree in Operational Research with Computing from Leeds University, England, in 1971, and the Ph. D. degree in Operations Research from Trinity College Dublin, Ireland, in 1975. In 1982 he joined the Department of Computer Science, North Carolina State University, as an Associate Professor, and since 1988 he is a Professor. His current research interests are in the area of ATM networks and their performance. He is the chairman of the IFIP WG 6. 3 on "Performance of Communications Systems".

Ramon Puigjaner received the degree of Industrial Engineer from the Polytechnic University of Catalonia (Spain) in 1964, his Master degree in Aeronautical Sciences from the Ecole Nationale Superieure de l'Aéronautique de Paris (France), his Ph. D. from the Polytechnic University of Catalonia (Spain) in 1972 and his degree of License in Informatics from the Polytechnic University of Madrid (Spain). He is member of the IFIP WG 6.3 on "Performance of Computer Networks", WG 6. 4 on "Local and Metropolitan Communication Systems" and of the WG 10. 3 on "Distributed Systems". His current research interests are the performance evaluation of computer systems and its application to industrial milieu. 